\title{
Adopting and Applying Eco-Design Techniques: A Practitioners Perspective
}

\author{
Paul Knight ${ }^{a^{*}}$, James O. Jenkins ${ }^{b}$, \\ ${ }^{a}$ Research and Development Department, Smiths Detection - Watford Ltd., Park \\ Avenue, Bushey, Watford, Hertfordshire, WD23 2BW. \\ ${ }^{\mathrm{b}}$ School of Life Sciences, University of Hertfordshire, College Lane, Hatfield, \\ Hertfordshire, AL10 9AB.
}

\begin{abstract}
This paper discusses the findings of a small scale research project which explored the possibility of adopting eco-design techniques. The paper focuses on identifying how eco-design techniques can be determined as being compatible with new product development processes. Via the development of a five stage 'applicability framework', this study demonstrates how a compatible suite of tools can be identified for application to product development processes. Testing and validation of this 'applicability framework', which was used to identify three key eco-design techniques; namely checklists, guidelines, and a material, energy and toxicity (MET) matrix, is shown to have taken place in relation to the development of a lightweight chemical detector product. It is established that checklists, guidelines and the MET matrix can be used both on a specific product, and also more generally in the design process. In particular, the MET matrix is shown as being used to successfully identify key environmental aspects of the product during its lifetime. The paper concludes by arguing that eco-design techniques may not have been more widely adopted by businesses because such methods are not necessarily generic and immediately applicable, but instead require some form of process-specific customisation prior to use, which can in turn act as a barrier to adoption . It is also highlighted that the shear diversity of pressures that come to bear during the product development process can also act as a barrier to adoption, and that the full integration of eco-design techniques will have to encompass approaches which overcome such pressures.
\end{abstract}

Key Words: Eco-design tools, applicability framework, process-specific customisation, Design for environment, Eco-efficiency, product development

\footnotetext{
* Corresponding author

E-mail addresses: paul.knight@smithsdetection.com (P. Knight), j.o.jenkins@ herts.ac.uk (J. O. Jenkins).
} 


\section{Introduction}

This paper discusses the adoption of eco-design techniques and how their applicability can be determined in relation to new product development processes. In particular, the compatibility of eco-design techniques with the existing design process is established through development of an 'applicability framework' which has been used to identify three key tools; namely checklists, guidelines, and a material, energy and toxicity (MET) matrix. Testing and validation of this selected suite of tools is then shown to have taken place in relation to the development of a lightweight chemical detector product manufactured by Smiths Detection, a medium-sized international manufacturing business.

One way in which manufacturing industry can reduce the impact it has on the environment is for it to adopt 'eco-efficiency' approaches. In particular, 'eco-design' is increasingly viewed as being key to sustainable and improved product development ([1], p.48). However, if eco-design approaches are to be effective, and have a significant impact, the chosen approaches need to be based on sound design and engineering principles that are accessible and lend themselves to being supported throughout the design and manufacturing process ([2], p.15). Despite the apparent benefits of eco-design, "it is unclear if these tools are being used and if they have any real effect on product system developments" ([3], p.1396-1397). Indeed, research by Baumann et al. [4] and Schishke et al. [5] indicates that application of eco-design tools and methods by small and medium sized enterprises is limited. In addition to discussing the application of eco-design methodology, and the applicability framework developed during the course of this study, this paper concludes by arguing that eco-design techniques may not have been more widely adopted by businesses 
because such methods are not necessarily generic and immediately applicable, but instead must include some form of process-specific customisation prior to use. This in turn, can act as a barrier to adoption.

To explore the usefulness of the developed 'applicability framework' this paper is split into six subsequent sections. The first section provides an overview of this case study; the second discusses the chosen methodology; the third describes how applicability was assessed; the fourth discusses application of eco-design theory in the design process; the fifth discusses the applicability framework and the potential for its more general use, and reflects on this case study from a practitioner's perspective; and conclusions are drawn and recommendations made, in the final section.

\section{A brief overview of the study context and research rationale}

Manufacturing industry has been accused of operating a system that takes, makes and wastes, although it also has the potential to become a creator of products that generate ecological, social and economic value [6]. One possible way to improve on this viewpoint is for industry to embrace the 'eco-efficiency' approaches. In the specific case of the design process, this might involve the adoption of 'Design for Environment' or 'Eco-design' techniques.

Although traditionally, the design process itself consumes few resources, about 15 per cent of manufacturing costs, it is responsible for committing the remaining 85 per cent. In a wider context, design might be considered responsible for most, if not all, environmental impacts ([7], p.9). This issue is often overlooked when impacts are considered during the development of Environmental Management Systems (EMS) 
as: "Products are seldom regarded as significant aspects and are therefore not within the main scope of many EMSs" ([8], p. 417). Put another way, “...the linkage between EMSs and product development is weak or completely missing” ([9], p. 377). Eco-design seeks to redress the balance, and make the necessary links, by recognising this impact of the broad role of design, through a process which addresses the wider picture (Figure 1).

\section{Figure 1: Eco-design: the wider picture - to go here}

Sometimes known as 'Design for the Environment' (DfE), eco-design has been defined as "the systematic integration of environmental considerations into product and process design" ([11], p. 1). The process aims to minimise the costs and "adverse environmental impacts of products throughout their entire life cycles" (ISO/TR14062, clause 4) [12]. There are limits however. It should be remembered that eco-design only adds environmental considerations to product design, it stops short of full sustainable design. Such an approach would incorporate more innovative practices, employ ecological principles, and encompass social and ethical aspects ([7], p.13).

Understanding of what eco-design actually is has now developed to the point where it has recently been described as "not a specific method or tool" but rather a "way of thinking and analysing..." ([13], p. 3). In practice then, this way of thinking and analysing, takes the various eco-design methods, as might be appropriate, and applies them to each of the different phases (or levels ) of the design process (see Table 1). In order to be effective though, the chosen methods need to be based on a sound foundation in design and engineering "...that is also integrated with the 
environmental sciences" ([14], p. 1292). And then, if eco-design is to make a truly significant impact, the "systematic integration" issues will also need to be addressed, so that EMSs can fully encompass product development, and be effective over the longer term ([15], p.405). This will need a strategic approach and, of necessity, involve development of not just those within the organisation but will also require participation throughout the entire production and consumption chain ([16] p.247).

\section{Table 1: The Four Levels of Design - to go here}

Despite the apparent benefits of eco-design, most notably; maintaining competitiveness, reducing production costs, identifying new opportunities and improving regulatory relationships [18], evidence of actual implementation is sparse: "The literature is full of examples of pilot DfE projects on the corporate level, but of few examples of the introduction into product development" ([19], p.18). Other researchers have noted in particular that SMEs rarely implement eco-design in the product development process ([5], p.235).

In conclusion, the reasons for the lack of adoption are unclear. This study sought in part to address this question, and may in turn help to explain why eco-design has not been more broadly adopted as a routine process within new product development. Also, eco-design tools are currently viewed as 'tools for experts' - in order to be made useful, they need further interpretation for an individual company's business sector and product type ([20], p. 674). By development of an 'applicability framework', this study seeks to demonstrate how a compatible suite of tools can be identified for application to the product development process. 


\section{Methodological Overview}

The development of the 'applicability framework' for eco-design techniques took place in the context of Smiths Detection - Watford Ltd. (SDW), a light manufacturing company which is involved in the design and manufacture of gas and vapour detection equipment for use by military and security customers. The following methodological overview is split into five sub-sections that firstly focus on justifying the company selected for the study, and then how appropriate eco-design tools and techniques were identified. This is followed by a section that outlines how the product development process at SDW was reviewed to identify potential areas suitable for adoption of the tools. The fourth sub-section focuses on detailing how compatible eco-design tools were then identified as being appropriate for usage. Finally, the methodology overview focuses on discussing how the theory was applied and how the selected eco-design tools were validated as being useful.

\subsection{Company selection}

The company was deemed suitable for the proposed research because it had expressed a specific interest in eco-design (by taking part in a 'demonstrator' project [21]) but had not followed it up. It is not clear why this is the case, but it was believed that ecodesign could be adopted, if the 'right' tools were identified to facilitate uptake of the process. Also, new product development represents a very significant part of the company's business thus making it a prime target for the adoption of eco-design techniques.

The research, design, and development process, known internally as 'New Product Introduction' (NPI), has been developed over many years. It now consists of a 'gated' 
system where certain criteria have to be met prior to moving from one 'phase' to the next. This approach lends itself naturally to development and adoption of environmental criteria at appropriate gates.

\subsection{Identification of eco-design tools and techniques}

The project started with a bibliographic review of current published material on ecodesign tools, techniques and case studies. Sources included: peer-reviewed journals, books, internet sites, international standards and government and SDW documents. This literature search helped to ensure that suitable guidance on identifying ecodesign tool typologies and criteria for tool assessment was obtained, and that all lifecycle issues were addressed.

During the course of the research, a range of tools and techniques were identified that would benefit from formal categorisation, if they were to be of use. Calow et al. [10] suggest just two categories: qualitative and quantitative, that can be used to categorise these tools. This simple idea, together with the need to use appropriate methods and language that would be recognised by the end-users, prompted the development of the following three broad categories for use in this project:

1 Guidelines: defined here as: providing broad support, with little detail, but applicable either across the whole product development process and lifecycle, e.g. ISO/TR 14062, or covering a significant area, e.g. design for recycling; design for disassembly; design for lifetime optimisation (see for example [2225]). 
2 Checklists: defined here as: providing in-depth, but narrow, application at selected stages of the product development process or lifecycle (see for example [26-28]).

3. Analytical tools: defined here as: providing detailed and/or systematic analysis at specific stages of either the product development process or lifecycle e.g. eco-indicators; environmental effect analysis; environmental impact assessment; life cycle assessment; material, energy and toxicity ('MET') matrix; life cycle cost analysis (see for example [29-34]).

It has been observed that: "DfE methods and tools must not only be appropriate from an environmental perspective, but from a user perspective as well” ([35], p. 228). So, in order to understand the users' perspectives, it was necessary to understand the potential application of the tools. This aspect is examined in the next section.

\subsection{Investigation into the product development process at Smiths Detection}

As a comprehensive understanding of the 'state-of-the-art' of eco-design was developed, the project also considered current company practice by reviewing existing SDW product design and development processes ('NPI') on a stage-by-stage basis. Using the above guidance on eco-design tool typologies and criteria, and qualitative research methods, i.e. by "asking questions and making comparisons" ([36], p. 132), some likely process areas, e.g. specification, concept, and design review activities, were identified at an early stage in the project as being suitable for application of the tools. 
Judgements were then made on: feasibility of application, possible gains/costs/benefits and the potential for systematic integration, as described in the next sub-section.

\subsection{Identification, analysis and review of compatible eco-design tools}

As part of the content analysis, key features such as advantages and disadvantages were considered, together with other relevant aspects which became apparent, and further consideration was given to how they might address such aspects as: resource consumption minimisation, packaging, the potential for recycling and the identification of alternatives to hazardous materials. Other aspects to address, which were identified included the impact on:

- $\quad$ materials - e.g. the use of lead-free solder;

- $\quad$ energy - e.g. as used in the routine baking process;

- $\quad$ waste - e.g. as generated by the manufacturing and in-use processes; and

- water - e.g. as used in the routine component cleaning process,

Particular reference was made to the ISO Technical Report ISO/TR 14062 [12]. This document describes the process of integrating environmental aspects into product design and development, and may become an international standard at some future date - and so is thought to be a key document in this analysis. It was also included as a 'tool' in its own right. 
In making a judgement on the appropriateness of the methods and tools identified above, and in order to draw up a short-list, questions derived from best-practice guidance were used as criteria for evaluation, as follows:

- $\quad$ Does the tool support the meeting of product requirements?

- Does the tool address environmental product legislation requirements?

- $\quad$ Can the tool be used by company staff, or will external expertise be required?

- Does it reduce the risk of important elements being forgotten?

- Does it fit in well with how products are launched by the company?

- Is the tool user friendly, easy to adopt and implement?

- Does it save time in the overall development process?

- Is it affordable by the company?

(adapted from: $[35,37])$

ISO/TR 14062 suggests possible actions, related to environmental aspects, to be taken at each stage of the product development process. Using that report as a framework, the above questions have been asked of each phase of the SDW NPI process to establish the level of appropriateness of each tool and to assist with drawing up a short-list.

At this stage, use was made of a focus group (a method often used to evaluate new product ideas ), in order to review the short-list. This approach was considered to be an efficient use of the time available - as a result of the interactive discussion which takes place [38], and also because “...synergy between the discussion contributors 
provides data through sharing and comparing knowledge, rather than individual questioning” ([39], p. 436).

Invitations were sent to a group of 12 engineers, scientists and managers, selected on the basis of their competence in the area of new product development. This included a request for those unable to attend to select a suitable delegate, if possible, so that the group size would eventually be near optimum - "usually 8-10" ([38], p. 189). As diverse a range of staff as possible was included, within the limits of ensuring competence, so that views from all levels of the organisation could be ascertained. They were asked to discuss the relative merits of the proposed techniques and their applicability to the 'real world' at SDW, based on their personal experiences of developing the company's products. Their remit being to express opinion on how the tools might fit into the NPI process. The ranked views collated from the focus group would effectively identify the most promising techniques for testing on a product development.

\subsection{Application of eco-design theory to the product development process}

Prior to testing, the tools underwent a degree of 'translation', as the "common language" of the company is needed if implementation of eco-design is to be successful ([40], p. 307) and they also need to be "compatible with the culture and current systems at a company" ([41], p. 3). This was accomplished with support from selected members of the focus group: a small multi-disciplinary team was formed which included: mechanical, electrical / electronic, materials and environmental expertise. They were asked to review the selected tools in more detail and offer suggestions for enhancement of the text used. 
The choice of a product was then made. The selection was based on relevant criteria, drawn from best-practice examples ([42], p. 63), in order of relevance to the business, as follows:

1. organisational capacity and financial resources;

2. estimated market potential and income generation;

3. the necessity to reduce the product's environmental impact based on the company's environmental policy, legislation and customer requirements;

4. potential for the development team to overcome environmental problems;

5. anticipated learning effect for the whole organisation;

6. complexity of the product; and

7. possibility of combining innovation with environmental merit

It had been thought that either the 'Lightweight Chemical Detector' or the 'BioSeeq' products might be suitable for this work, both are subject to on-going development, with potential for environmental impacts. Both are complex and have long life cycles: where this is the case, it is recommended that just one component or sub-assembly of a product be used for evaluation ([42], p.65). It would therefore be necessary to identify specific aspects of the development that could be addressed using the chosen tools. This was discussed with the team derived from the focus group, and areas for attention were agreed. 


\section{Assessing the applicability of eco-design tools and techniques}

With the better understanding of the available range of eco-design tools, which had been developed during the categorisation exercise, and knowledge of the possible process areas where they might be applied, appropriateness of each tool was judged on the basis of the eight questions listed previously. To simplify this analysis, and prior to the assessment, a number of tools were grouped together, as follows:

- $\quad$ Most of the guidelines identified are encompassed by the BSI and ISO documents (included as tools in their own right), 'Guidelines' are therefore treated as a single tool.

- $\quad$ 'Restricted Material Lists' are regarded as a 'Checklist', so these are also included in this single tool.

- Eco-indicators were of limited use on their own but, when grouped with the material, energy and toxicity ('MET') matrix, had the potential to make that tool more useful, so are included there.

In order to identify suitable tools for consideration by the focus group, simple yes / no responses were made to the criteria derived from best practice guidance. The tools were then listed in order of preference as measured by the number of 'yes' answers. The results are shown in summary form in Figure 2.

Figure 2: Initial analysis of identified eco-design tools - to go here 
The authors identified their short-list of tools for further consideration based on a score of five or greater. Those scoring less than five were not considered further. It should be emphasised that, at this stage, the grading of the appropriateness of the tools is only based on the opinion of the author. Prior to presenting this short-list to the focus group, the above ranking information was removed and the order of the tools was randomised so that the group was not unduly influenced by the authors' views.

Following a brief presentation on the rationale behind each of the tools, ensuing discussion included the following key comments:-

- $\quad$ The Lifecycle Development Strategy Wheel (LiDS) and other strategy tools would usually be over-ruled by customer specifications - development contracts now usually include specific product environmental impact requirements;

- disassembly and recycling are considered to have lower priority compared to longevity issues - the company routinely designs for long life, then carries out repair and overhaul activities on in-service products, usually after their initial 15 year life, effectively extending the actual product lifetime to more than 20 years.

- the "10 Golden Rules" represent common sense - "we do it anyway". Also, they lack specificity and need more detail;

- "checklists need to be easy to follow" - include in design review; 
- $\quad$ sample eco-indicators look promising - need more;

- $\quad$ concern over resource impact (i.e. staff time) from 'Environmental quality function deployment' tool - only useful if the specification is flexible

- "LCA is too complex, and not user friendly"

The group session concluded by ranking the techniques in order of preference. Group members were asked to complete a form to confirm their individual opinions on the relative applicability of each tool to the company's design practice. The results are shown in summary form in Table 2. Given the limited amount of time for further work, it was decided that the first three preferences would be carried forward to the next stage for validation. Assuming a possible 50 per cent failure rate in the validation process, this would still result in one or two tools being declared to be compatible with the NPI process.

The initial analysis (as shown in Figure 2) had revealed a range of applicability of the 15 tools under consideration. The focus group then identified the tools that could be used, and those that might be more difficult to apply. In particular, management tools, for example, were considered of limited use, as tools need to fit into the current, tested (and trusted) structure. In particular it was considered that strategy tools, e.g. the 'LiDS wheel', would usually be over-ruled by customer specifications, thus implying a certain lack of freedom in (eco) design. The group considered that it would be more effective to focus on technical guides and analytical tools instead. 
Table 2: Focus Group tool ranking results - to go here

Disassembly and recycling methods were viewed as having a lower priority, compared with longevity issues, and were not seen to be immediately relevant. There is a growing awareness that this view might also apply to the consumer market, where lifetime issues are beginning to be more widely recognised as an issue [43]. It is anticipated that further work might be published on this particular aspect in the future. For the moment SDW undertakes repair and overhaul (R\&O) activities on existing products and considers 'technology insertion' approaches in new product development to allow for in-service upgrades to keep the product(s) current and up-todate, thus extending lifetime.

Although ranked ninth by the focus group, i.e. least preferred, this discussion would not be complete without mention of life cycle analysis. It has been described as the "scientific response" ([44], p. 141) to the need to understand the environmental impact of products and has a key strength in its quantitative approach ([45], p. 30). While this may be true, the technique requires a very significant investment in time and effort, and is "...not useful in the design process... companies cannot delay their design process to wait for the results..." ([46], p. 8/2). In fact, it has been described as “...an improper method for product development” ([47], p. 494). The focus group considered it to be too complex and not user friendly and so effectively rejected it. Nonetheless, the tool of choice, the MET matrix, does address all parts of the product lifecycle and is therefore believed to be sufficient to meet the needs of the corporate policy requirement, at least for the moment. 


\section{Application of eco-design theory to the product development process}

Before any validation of the tools could be carried out, choice of a suitable product was necessary. Selection was made based on the criteria detailed earlier.

Both the 'Lightweight Chemical Detector' and the 'BioSeeq' product had initially been identified as possible candidates. The market potential (criterion 2) for the Lightweight Chemical Detector was noted as being very large: as a personal detector, it is designed to be issued to individuals - whereas other detectors, including the BioSeeq, are generally intended for group protection and are consequently produced in fewer numbers. Sales volumes are difficult to predict for such devices, but it is confidently expected that the Lightweight Chemical Detector product will be made in many 1000s. The consequent environmental impact is therefore worthy of consideration (criterion 3), and so the Lightweight Chemical Detector was chosen to test the selected tools.

Further support for this choice, in terms of organisational capacity (criterion 1), came about as a result of a timely customer order [48]. A quantity of 450 lightweight chemical detectors was ordered for the Swedish defence forces, and the contract included a requirement to assess the environmental impact of these units. This allowed the personnel, to be made available to carry out the work.

\subsection{Application of Checklists}

The need for translation into the common language of the company was accomplished with support from the small multi-disciplinary team selected from the focus group. They were asked to review the sample checklists [26, 27], with a view to preparing a set suitable for use in the SDW NPI process. Despite the product's complexity, and 
consequent potential difficulty in addressing all appropriate issues at once (identified earlier), it was considered possible to generate checklists which could be used at most of the stages of the NPI process, as Figure 3 serves to exemplify. Other checklists were developed for initial system design: in particular, to aid design of electronic assemblies and to assist with identifying later disassembly and recycling potential. Indeed, the development and use of checklists were recognised by the whole group as being useful, and more widely, they are seen as being "easy to understand, and are often the first tool a company starts to use when getting into eco-design" ([37], p. 15).

\section{Figure 3: Sample checklist - to go here}

\subsection{Application of Guidelines}

Suitable guidelines had been identified as part of the content analysis procedure. They included:

- guidance on integrating environmental aspects into product design and development - ISO/TR 14062 [12];

- design for manufacture, assembly, disassembly and end-of-life processing (MADE) - BS 8887-1 [49];

- $\quad$ sustainable design of electrical and electronic products to control costs and comply with legislation - GG427 [50]; and

- Smiths Group Eco-Design Guide [28].

The group agreed that these were immediately usable and should be included in the design process; although the literature suggests that some caution should be applied. 
For example, adoption of Design for Disassembly (DfD) might best be limited to major sub-assemblies and for re-use applications [51]. It can help eliminate the potentially most hazardous part of the supply chain, but "harsh use" [52] can lead to premature disassembly and radical ideas like water soluble screws need to be treated with considerable caution - especially in military applications. In addition: “...studies have shown that little or no added value is created by designing products for disassembly..." (at least for recycling purposes) and Design for Nondisassembly (DfND) is advocated by some ([53], p. 219), as materials separation technology has improved dramatically in recent years. The priority for SDW is design for longevity and this may ultimately obviate the need for DfD considerations in the design process, at least in the short to medium term.

\subsection{Application of The 'MET' matrix}

The Material, Energy and Toxicity ('MET') Matrix is a tool which can be used to summarise the environmental impact at each stage of a product's lifecycle. The use of this matrix to assess the Lightweight Chemical Detector product highlighted the production process as having the most significant impacts: the high use of energy, and the effluent volumes resulting from the need for scrupulous cleaning of component parts during the manufacturing process. These aspects would benefit from closer examination, as eco-efficiency improvements in both these areas might also generate significant cost savings. These, and other findings, are summarised in Table 3.

Table 3: 'MET matrix' for Lightweight Chemical Detector product - to go here

The MET matrix could be enhanced by the use of eco-indicators to provide detailed, and quantified, analysis of the environmental impacts of the new product. Weight data 
for individual components is available from the company's three-dimensional computer aided design (3D CAD) system, and the system's supplier has been contacted to see if eco-indicators could be included as part of their software package. The initial interest is positive - it may in fact generate market interest for them as part of their own product development.

\section{Discussion}

It has been suggested that researchers in this field need to investigate product development processes to identify how "environmental concerns... can be translated into product specifications" ([54], p. 282) and to consider both the operational and the management levels [55]. This is the approach which has been adopted here. It is encompassed by the 'applicability framework' concept which has been developed.

\subsection{The Applicability Framework}

The tools determined for use in the eco-design procedure at SDW have been identified and assessed using the methods described above, but it is considered worthwhile summarising the approach in more general terms, as this may prove useful in other organisations. It is possible to identify five distinct stages (as summarised in Figure 4):-

1. Investigation into available tools and into the specific design process under consideration - so that both are well understood (in the case of this study: a thorough literature search and review of the company's design process), followed by 
2. Compatibility Analysis (in the case of this study: by use of the criteria listed above as an initial assessment, in order to develop a short-list of tools), then refined through

3. Compatibility Peer Review (in the case of this study: a focus group discussion on the short-listed tools by a range of competent staff representing all levels of the organisation), followed by

4. Adaptation and refinement of the most promising tools identified by the peer review (in the case of this study: by a specialist team, derived from the focus group, comprising staff who are also directly involved in the design process on a daily basis), and finally

5. Validation through compatibility confirmation on a sample product, and subsequent incorporation into standard company processes (in the case of this study: application of the MET matrix to the Lightweight Chemical Detector product using the guidance and checklists identified earlier, and modification of the NPI gate checklists to incorporate these tools as an eco-design 'procedure').

The essence of this approach to establishing applicability can simply be expressed as follows:-

$$
\text { Applicability }=\text { Compatibility }+ \text { Adaptation }+ \text { Validation }
$$

Figure 4: The Applicability Framework - to go here 


\subsection{The Practitioner's Perspective}

The diversity of approaches toward eco-design reported in the literature led to some initial difficulties in organising the data. This is not a unique experience: other researchers have categorised eco-design tools in many different ways, with few common features in their work, although 'analysis / assessment' and 'improvement / priorities' classifications appear most frequently. As a consequence, a simple categorisation method proved to be the inspiration for the approach adopted in this study (see [10]). Three broad, but potentially useful, categories were subsequently identified: checklists, guidelines and analytical tools.

In general, software tools (either standalone or web-based) implement one or more of the tools included in this study - but in combination. They were therefore not considered immediately suited to the SDW NPI process. However, although beyond the scope of this study, it is recognised that they may be beneficial, at a later stage, for simplifying the use of the more complex methods such as life cycle assessment.

The Lightweight Chemical Detector product study showed that checklists, guidelines and the MET matrix can be used both on a specific product, and also more generally in the design process. In particular, the MET matrix was used successfully to identify key environmental aspects of the product during its lifetime, and design process issues were supported by the checklists and guidelines.

In a broader context it seems that the motivating factors behind this project are similar to the external stimuli affecting other organisations, i.e. customer, government and corporate interest (see [56]). Internal factors differ however: the main motivation for 
SDW comes from the need for continual improvement to support the EMS and to reduce waste and energy costs, rather than an aspiration to innovate, improve quality, and address new markets. "...eco-design ... only stands a chance, if it is supported by stimuli other than the environmental benefit alone" ([57], p. 453), so cost savings may prove to be the key to success or failure of implementation in the longer term.

In the context of this study, it would appear that the six success factors identified in this area by Johansson [55] have been successfully addressed: i.e. consideration of environmental issues at the beginning of product development; integration into the existing development process; checkpoints, review and milestone questions introduced into the process; company-specific design principles, rules and standards used; use made of cross-functional teams, and application of support tools. Although, it is recognised that broader issues still need to be considered, e.g. customer and supplier relationships.

In retrospect, success has been achieved (so far) in part because the approach used in this study resulted in a choice of flexible tools with which design staff could readily empathise - checklists, for example, were already used in the NPI process to support design reviews. Although, addressing a product's life cycle via a checklist could be considered by some to not necessarily be the same as conducting a Life Cycle Assessment (which could identify potential trade-offs when changes are made to a system). For example, a checklist might encourage 'recycling' but not instruct the user to investigate the potential consequences. However, we contend that a checklist is appropriate in this context because it has helped to establish a (basic) eco-design procedure that is compatible with, and now integrated within, company processes and 
is sufficiently generic to be flexible enough to be applied to a number of different company products. Guidelines are also familiar as reference material, yet flexible, and are used elsewhere throughout the existing processes in support of detailed engineering design activity - on sealing, ruggedisation and chemical resistance, for example. And finally, the MET matrix is comparable to similar tools used to ensure compatibility with customer contractual requirements, where a compliance matrix is used on a routine basis.

These tools, and particularly the checklists, have undergone review and adaptation prior to use by SDW, without which they would have been far less acceptable to the design staff. It is of note that further investigation has, for instance, identified ecoindicators as a means to enhance the MET matrix and encourage deeper consideration of potential consequences, and will supplement the designers' existing technical skills and knowledge in the use of this particular tool. This latter point is also an example of how the tools can, and may need to be, adapted for use within a specific application. Acceptability by, and usefulness to, the design staff are crucial attributes of the tools if they are to be adopted successfully: the staff's involvement in this process has not only assisted in the choice of tools, but has also ensured their acceptability to the design community - and consequently enhanced the chances of successful long-term integration.

'Usefulness' also extends to the overall process: if products are to be designed for long service life, then 'Design for Longevity' is paramount to the business, as the focus group identified here. In the consumer market, the opposite may be true, as product lifetimes are often very short - perhaps only a matter of months: in that case, 
Design for Re-use/Recycling would be far more important. The impact of this observation is to reduce the relative importance of other approaches, in this particular study - Design for Recycling (at least in the short term), and represents a further example of how customisation of a suite of tools to build an eco-design procedure can make for a successful approach. Ultimately, market and customer demands, as well as the motivating factors mentioned earlier, and legislative requirements, will all have a bearing on the final choice.

It became clear during the focus group discussions that the current design process (NPI) is very much trusted by the company's design community - any proposed change is viewed with a healthy scepticism. It was also pointed out that many other pressures come to bear during the product development process and that staff need to make efficient use of the available time. This view has been succinctly illustrated by Luttropp and Lagerstedt in their product development 'cake', as Figure 5 shows. So full integration represents a significant challenge, and if this is to be achieved, then further work will be required.

Figure 5: The Product Development 'cake' - to go here

It has been recommended that: more products are used to develop the identified tools, more checklists are developed in order to encompass the full NPI process, and ecoindicators be introduced to enhance use of the MET matrix. In addition, consideration should be given to Environmental Effects Analysis, the fourth-rated tool by the focus group, as this shows potential, and is similar to Failure Mode and Effect Analysis - a QMS technique which is well established at the company. It is also recognised that 
the eco-design procedure will need to draw upon detailed information in order to support the making of design decisions. Some sources have been identified; the 3D CAD system for material useage (weight) data; eco-indicators as an overall figure of merit for material environmental impact; material safety data sheets (MSDS's) for toxicity information; and manufacturing process data (for energy and water useage)). But more will be required: as noted earlier, supplier relationships need development and they may then assist in the flow of information on components, materials and their possible alternatives. In doing so, this initial implementation should then lead to full integration.

\section{Conclusions}

The main project objectives have been met; compatible tools have been identified, validated, and integrated into a basic eco-design procedure within the overall design process. However, full integration will have to encompass approaches which overcome the remaining perceived barriers. In particular, it is of note that the focus group highlighted that:

- $\quad$ strategy tools would usually be over-ruled by customer specifications, implying a lack of freedom in applying eco-design and restricting the company's scope to implement a self-determined strategy. So the process should work within these limitations and omit such tools, at least until an 'eco-design culture' is well established;

- some tools are more appropriate than others (in this case, longevity takes priority 
over disassembly and recycling, for example). So the procedure needs to adopt only process-compatible tools that show clear benefits and include only those for which staff are prepared to take responsibility [see 56];

- some tools represent common sense (e.g. the "10 Golden Rules" - "we do it anyway"), but lack specificity. So where they are already inherent in the design process they need recognition and development;

- ease of use, complexity, and resource impact (i.e. staff time) are common themes, and to some extent, these factors are inter-dependent. The need for staff training and development has been identified and should ease concerns in these areas;

- other pressures come to bear during the product development process. This may be the greatest obstacle, as time is always a limited design resource.

In general then, clear, and visible, adoption of tools accepted as being useable by, and useful for, the design community should lead to full acceptance in the longer term.

As for general adoption across industry, it is the authors' view that the range of approaches described in the literature serves to add to the challenge, rather than help meet it. As noted earlier, with so many other aspects already to be considered during a design project such diversity only adds to the workload of adoption.

It is not therefore surprising then, with such a wide range of techniques, that not all are found suitable for use in a particular design process application. This suggests 
another possible reason for the lack of widespread adoption: there is no one-size-fitsall solution, and some form of process-specific customisation is needed in each case, if the techniques are to be widely implemented. This can be addressed at two levels: firstly, choice of the tools which will ultimately support the eco-design procedure that is to be implemented, and secondly, the detailed adaptation of those tools to the specific needs of the design process under consideration. The applicability framework which has been described here is offered as a possible means to that end.

In this example, the tools and particularly the checklists, have undergone review and adaptation prior to use by SDW. Without this review process they would have been far less acceptable to the design staff. This study has resulted in an eco-design procedure with which they can readily empathise. The earlier work carried out at the company (i.e. the demonstration project) had brought in specialist expertise and had identified improvements, but the tools used at the time were not considered for compatibility. The demonstration project worked in isolation, and so the new techniques did not naturally become integrated in the design process, as has been achieved now.

Consequently, the work described here should lead to full integration, and go some way to addressing the 'takes, makes and wastes' accusation. McDonough and Braungart, ([6], p.62) have also suggested that "eco-efficiency only works to make the old, destructive system a bit less so" - they argue that more needs to be done and industry needs to go further. Eco-design, and ultimately sustainable design, will need broad adoption by industry if their concerns are to be fully addressed. Such a perception may have been true in 'conventional' industry, but there are definite signs 
that the manufacturing sector is changing, and more of an 'environmental' view is being taken.

This specific work, and subsequent further projects, will support that change. It represents an example of industry taking a broader, long-term, view of its responsibilities to society and to the planet, and serves to provide a tangible contribution to the overall aims of sustainable development.

\section{Acknowledgements}

The authors would like to thank the directors of Smiths Detection - Watford Ltd. for the provision of facilities and, in particular, Dave Wallace for his unstinting support throughout this project. In addition, they express their gratitude to John Brady for his editorial comments. Thanks are also expressed to Mary Ann Curran for her encouraging and pointed comments on an earlier draft of this paper.

\section{References}

[1] UK Parliament. Securing the future, delivering UK sustainable development strategy. CM6467. London: HMSO; 2005.

[2] Fitzgerald, D.P., Herrmann, J.W., Sandborn, P.A., Schmidt, L.C., and Gogoll, T.H. Design for Environment (DfE): Strategies, Practices, Guidelines, Methods, and Tools. In: Kutz, M., (ed). Environmentally Conscious Mechanical Design. London: Wiley; 2007. 
[3] Luttropp, C. and Lagerstedt, J. EcoDesign and The Ten Golden Rules: generic advice for merging environmental aspects into product development. The Journal of Cleaner Production 2006; 14: 1396-1408.

[4] Baumann, H., Boons, F., and Bragd, A. Mapping the green product development field: engineering, policy and business perspectives. Journal of Cleaner Production 2002; 10 (5): 409-425.

[5] Schischke, K., Mueller, J., and Reichl, H. EcoDesign in European Small and Medium Sized Enterprises of the Electrical and Electronics Sector. In: Proceedings of the IEEE International Symposium on Electronics and the Environment. Art. no. 1650067. 233-238; 2006.

[6] McDonough, W., and Braungart, M. Cradle to Cradle. Remaking the way we make things. New York: North Point Press; 2002.

[7] Tischner, U. Ecodesign in Practice. In: German Federal Environmental Agency Berlin (ed), How to do Ecodesign? A guide for environmentally and economically sound design. Frankfurt: Verlag form GmbH; 2000.

[8] Ammenberg, J., and Sundin, E. Products in environmental management systems: the role of auditors. Journal of Cleaner Production 2005; 13: 417431.

[9] Kautto, P. New Instruments - Old Practices ? The Implications of Environmental Management Systems and Extended Producer Responsibility for Design for the Environment. Business Strategy and the Environment. 2006; 15: 377-388.

[10] Calow, P., Holloway, L., and Owen, A. Ecodesign from the ground up: taking steps without footprints. A step by step guide to greener product development. Brighouse: Shot in the Dark; 2001. 
[11] NRC Canada. Design for Environment Guide. National Research Council, Canada. Available from: <http://dfe-sce.nrc-cnrc.gc.ca/home_e.html>; 2003 (read in November 2006).

[12] BSI. PD ISO/TR14062:2002, Environmental Management - Integrating environmental aspects into product design and development. London: British Standards Institution; 2002.

[13] Lindahl, M. Eco-Design - Practical lessons. Presentation given at Eco-design 06 Conference. $19^{\text {th }}$ June. Farnham: The Centre for Sustainable Design; 2006.

[14] Karlsson, R., and Luttropp, C. EcoDesign: what's happening? An overview of the subject area of EcoDesign and of the papers in this special issue. Journal of Cleaner Production 2006; 14: 1291-1298.

[15] Ammenberg, J., and Sundin, E. Products in environmental management systems: drivers, barriers and experiences. Journal of Cleaner Production 2005; 13: 405-415.

[16] Vermeulen, W.J.V., and Ras, P.J. The Challenge of Greening Global Product Chains: Meeting Both Ends. Sustainable Development 2005; 14: 245-256.

[17] Ferrendier, S., Mathieux, F., Rebitzer, G., Simon, M., and Froelich, D. EcoDesign Guide. Environmentally Improved Product Design Case Studies of the European Electrical and Electronics Industry. Report by the Ecolife Thematic Network, (Nov 1998 - Apr 2002); 2002.

[18] DEH. Product Innovation, The Green Advantage. An Introduction to Design for Environment for Australian Business. Department of the Environment and Heritage: Canberra; 2001.

[19] Ehrenfeld, J. and Lenox, M. J. The development and implementation of DfE programmes. The Journal of Sustainable Product Design 1997; 1: 17-27. 
[20] le Pochat, S., Bertoluci, G., and Froelich, D. Integrating ecodesign by conducting changes in SMEs. Journal of Cleaner Production 2007; 15: 671680.

[21] Turnbull, A. Delivering Advantage. Environment Business. March, 18-19. 2005.

[22] Knight, W., and Curtis. 'Design for Environment' software development. Journal of Sustainable Product Design 1999; 9: 36-44.

[23] van Nes, N., and Cramer, J. Design strategies for the lifetime optimisation of products. Journal of Sustainable Product Design 2003; 3: 101-107.

[24] Lofthouse, V., and Bhamra, T. End of Life Decisions - A Design Guide. In: Proceedings of the $11^{\text {th }}$ Annual International Sustainable Development Research Conference. Helsinki: University of Tampere; 2005.

[25] Herrmann, C., Frad, A., Luger, T., Krause, F-L., and Ragan, Z. Integrating End-of-Life Evaluation in Conceptual Design. Proceedings of the IEEE International Symposium on Electronics and the Environment. 8-11 May. Art. No. 1650069; 2006. p. 245-250.

[26] Clark, T., and Adams, G. Smart ecoDesign: Eco-design Checklist. For Electronic Manufacturers, ‘Systems Integrators', and Suppliers of Components and Sub-assemblies. Version 2. Farnham: Centre for Sustainable Design; 2002.

[27] ECMA. Environmental design considerations for ICT \& CE products. Standard: ECMA-341, $2^{\text {nd }}$ edition, December. Geneva: ECMA-International; 2004.

[28] Smiths. Smiths Group plc. Eco-Design Guide (Smiths Group internal document). Issue 1, December, 2004. 
[29] Tischner, U., and Dietz, B. The Toolbox: useful Tools for Ecodesign. In: German Federal Environmental Agency Berlin (ed), How to do Ecodesign ? A guide for environmentally and economically sound design. Frankfurt: Verlag form $\mathrm{GmbH} ; 2000$.

[30] Persson, J-G. Eco-indicators in product development. Proceedings of the Institution of Mechanical Engineers 2001; 215(5): 627-635.

[31] Tingstrom, J., and Karlsson, R. The relationship between environmental analyses and the dialogue process in product development. Journal of Cleaner Production 2006; 14: 1409-1419.

[32] Fuller, K. Environmental Impact Assessment. In: Brady, J., (ed). Environmental Management in Organisations. The IEMA Handbook. London; Earthscan; 2005.

[33] Boyd, S., Dornfeld, D., and Krishnan, N. Life Cycle Inventory of a CMOS Chip. Proceedings of the IEEE International Symposium on Electronics and the Environment. 8-11 May 2006. p. 253-257.

[34] BSI. BS EN ISO14040:2006, Environmental Management - Life cycle assessment - Principles and framework. London: British Standards Institution; 2006.

[35] Lindahl, M. Designers' Utilization of and Requirements on Design for Environment (DfE) Methods and Tools. Proceedings of the Fourth International Symposium on Environmentally Conscious Design and Inverse Manufacturing. Eco-Design 2005. Art. No. 1619207, pp.224-231. 2005.

[36] Strauss, A. L. and Corbin, J. Basics of Qualitative Research. Grounded Theory Procedures and Techniques. Newbury Park: Sage Publications; 1990. 
[37] Adams, G. Smart ecoDesign. Energy Using Devices (EuP) Eco-design Checklist for Electronic Manufacturers, System Integrators and Suppliers of Components and Sub-assemblies. Issue 1. Farnham: Centre for Sustainable Design; 2006.

[38] van Kleef, E., van Trijp, H.C.M., and Luning, P. Consumer research in the early stages of new product development: a critical review of methods and techniques. Food Quality and Preference 2005; 16: 181-201.

[39] Bruseberg, A., and McDonagh-Philp, D. New product development by eliciting user experience and aspirations. International Journal of HumanComputer Studies 2001; 55: 435-452.

[40] Pascual, O., Boks, C., and Stevels, A. Communicating Eco-Efficiency in Industrial Contexts: A framework for understanding the (lack) of success and applicability of Eco-Design. In: IEEE International Symposium on Electronics and the Environment 2003. p. 303-308.

[41] Kurk, F., and Eagan, P. The value of adding design-for-the-environment to pollution prevention assistance options. Journal of Cleaner Production 2007; 16 (6): $722-726$.

[42] Brezet, H., and van Hemel, C. Ecodesign: a promising approach to sustainable production and consumption. Paris, United Nations Environment Programme; 1997.

[43] van Nes, N., and Cramer, J. Influencing Product Lifetime Through Product Design. Business Strategy and the Environment 2005; 14: 286-299.

[44] Nuij, R. Environmental Product Development. In: Brady, J., (ed). Environmental Management in Organisations. The IEMA Handbook. London: Earthscan; 2005. 
[45] Everard, M. What goes around comes around. Magazine of the Institute of Environmental Management and Assessment. February 2005. p. 30-32.

[46] Bhamra, T.A., and Evans, S. An Ecodesign Model based on Industry Experience. In: IEE Seminar on: Engineering and the Environment - How it affects you! Ref: 1999/097. 8/1-8/4. 1999.

[47] Lindahl, M. Engineering designers' experience of design for environment methods and tools - Requirement definitions from an interview study. Journal of Cleaner Production. 2006; 14: 487-496.

[48] Smiths. Smiths Detection to help protect Swedish defence forces against chemical warfare agents. Press Release, 22 May. Available from: <http://www.smithsdetection.com/eng/1025_3006.php> 2007 (read in June 2007).

[49] BSI. BS 8887-1:2006, Design for manufacture, assembly, disassembly and end-of-life processing (MADE) - Part 1: General concepts, process and requirements. London: British Standards Institution; 2006.

[50] Envirowise. Sustainable design of electrical and electronic products to control costs and comply with legislation. Good Practice Guide GG427. Didcot: Envirowise; 2004.

[51] DTI. WEEE recovery: the European story. DTI Global Watch Mission Report, URN 06/1721. 2006.

[52] Russell, E. From cradle to cradle with active disassembly. Magazine of the Institute of Environmental Management and Assessment. October 2005. p. 1415.

[53] de Caluwe, N. Business Benefits From Applied EcoDesign. IEEE Transactions on Electronics Packaging Manufacturing 2004; 27(4): 215-220. 
[54] Berchicci, L., and Bodewes, W. Bridging Environmental Issues with New Product Development. Business Strategy and the Environment 2005; 14: 272285.

[55] Johansson, G. Success Factors for Integration of Ecodesign in Product Development: A Review of State-of-the-art. Environmental Management and Health 2002; 13(1): 98-107.

[56] van Hemel, C. What sustainable solutions do small and medium-sized enterprises prefer? In: Charter, M., and Tischner, U., (eds). Sustainable Solutions. Developing Products and Services for the Future. Sheffield: Greenleaf; 2001.

[57] van Hemel, C., and Cramer, J. Barriers and stimuli for ecodesign in SMEs. Journal of Cleaner Production 2002; 10: 439-453. 